\title{
THE MOLECULAR GAS CONTENT OF $z<0.1$ RADIO GALAXIES: LINKING THE ACTIVE GALACTIC NUCLEUS ACCRETION MODE TO HOST GALAXY PROPERTIES
}

\author{
V. SMOLČIĆ ${ }^{1,2,4}$ AND D. A. RIECHERs ${ }^{3,5}$ \\ ${ }^{1}$ European Southern Observatory, Karl-Schwarzschild-Strasse 2, D-85748 Garching b. München, Germany \\ ${ }^{2}$ Argelander Institute for Astronomy, Auf dem Hügel 71, Bonn D-53121, Germany \\ ${ }^{3}$ California Institute of Technology, MC 249-17, 1200 East California Boulevard, Pasadena, CA 91125, USA \\ Received 2010 December 13; accepted 2011 January 22; published 2011 March 4
}

\begin{abstract}
One of the main achievements in modern cosmology is the so-called unified model, which successfully describes most classes of active galactic nuclei (AGNs) within a single physical scheme. However, there is a particular class of radio-luminous AGNs that presently cannot be explained within this framework-the "low-excitation" radio AGN (LERAGN). Recently, a scenario has been put forward which predicts that LERAGNs and their regular "highexcitation" radio AGN (HERAGN) counterparts represent different (red sequence versus green valley) phases of galaxy evolution. These different evolutionary states are also expected to be reflected in their host galaxy properties, in particular their cold gas content. To test this, here we present $\mathrm{CO}(1 \rightarrow 0)$ observations toward a sample of 11 of these systems conducted with CARMA. Combining our observations with literature data, we derive molecular gas masses (or upper limits) for a complete, representative, sample of $21 z<0.1$ radio AGNs. Our results yield that HERAGNs on average have a factor of $\sim 7$ higher gas masses than LERAGNs. We also infer younger stellar ages, lower stellar, halo, and central supermassive black masses, as well as higher black hole accretion efficiencies in HERAGNs relative to LERAGNs. These findings support the idea that HERAGNs and LERAGNs form two physically distinct populations of galaxies that reflect different stages of massive galaxy buildup.
\end{abstract}

Key words: galaxies: active - radio continuum: galaxies

Online-only material: color figure

\section{INTRODUCTION}

Over the past two decades, a standard model of active galactic nuclei (AGNs) has emerged. In this "unified" model, efficient disk accretion of cold matter on the central supermassive black hole $(\mathrm{BH})$ provides the radiation field that photoionizes emission-line regions. However, there is a certain fraction of AGNs identified by radio observations that poses a challenge to the unified model, the so-called low-excitation radio AGN (hereafter LERAGN). The main difference between high-excitation radio AGNs (HERAGNs) and these LERAGNs is that the latter do not exhibit strong emission lines in their optical spectra (Jackson \& Rawlings 1997; Evans et al. 2006).

Recently, Hardcastle et al. (2006) have suggested that HERAGNs and LERAGNs may represent a principal separator between populations fundamentally different in their $\mathrm{BH}$ accretion mechanisms (see also Evans et al. 2006; Allen et al. 2006; Kewley et al. 2006). They developed a model in which central supermassive BHs of HERAGNs accrete in a standard (radiatively efficient) way from the cold phase of the intragalactic medium (IGM), while those of LERAGNs are powered in a radiatively inefficient manner by Bondi accretion of the hot IGM. Smolčić (2009) showed that LERAGNs and HERAGNs exhibit not only systemic differences in their BH masses and accretion rate properties but also in their host galaxy properties, such as stellar masses and stellar populations. This is consistent with these two classes of radio AGNs dividing in a stellar mass versus color plane in such a way that LERAGNs occupy the red sequence and HERAGNs inhabit the so-called green valley, a

\footnotetext{
4 ESO ALMA COFUND Fellow.

5 Hubble Fellow.
}

sparsely populated region between the blue cloud and the red sequence (Smolčić 2009).

The stellar mass versus color plane can be interpreted as a time sequence for galaxy evolution. Galaxies are thought to evolve from an initial star-formation-dominated state with blue optical colors into the most massive red-and-dead galaxies through a transition phase reflected in the green valley (Bell et al. 2004a, 2004b; Borch et al. 2006; Faber et al. 2007; Brown et al. 2007). In recent years, it has been suggested that radio outflows from AGNs likely play a crucial role in this massive galaxy buildup (Croton et al. 2006; Bower et al. 2006; Sijacki \& Springel 2006; Sijacki et al. 2007). In this context, the radio-AGN feedback (often called the "radio" or "maintenance" mode), which is thought to limit stellar mass growth in already massive galaxies, is expected to occur only in LERAGN (Smolčić 2009).

Furthermore, it has been shown that the cosmic evolution of the space density of various types of radio AGNs is significantly different (e.g., Peacock 1985; Willott et al. 2001; Smolčić et al. 2009). Based on a study of the evolution of the radio AGN luminosity function out to $z=1.3$, Smolčić et al. (2009) have shown that the comoving space density of low-luminosity radio AGNs (predominantly LERAGNs) only modestly declines since $z=1.3$, while that of powerful AGNs (predominantly HERAGNs) dramatically diminishes over the same cosmic time interval. This suggests that LERAGNs and HERAGNs not only represent physically distinct galaxy populations but also populations in different stages of massive galaxy buildup. If this is the case, the molecular gas masses and fractions in LERAGNs and HERAGNs are expected to directly reflect this trend.

We here investigate this idea by observing $\operatorname{CO}(J=1 \rightarrow 0)$ emission of a carefully selected, representative sample of nearby $(z<0.1)$ HERAGNs and LERAGNs with Combined Array 
Table 1

Physical Properties of the $z<0.1$ Sample of Radio AGNs

\begin{tabular}{|c|c|c|c|c|c|c|c|c|}
\hline Name & Redshift & Type & $\begin{array}{c}L_{178 \mathrm{MHz}} \\
\left(\mathrm{W} \mathrm{Hz}^{-1} \mathrm{sr}^{-1}\right)\end{array}$ & $L_{0.5-10 \mathrm{keV}} / L_{\mathrm{EDD}}$ & $\begin{array}{l}\text { Stellar Age } \\
\text { (Gyr) }\end{array}$ & $\begin{array}{c}M_{*} \\
\left(M_{\odot}\right)\end{array}$ & $\begin{array}{l}M_{\mathrm{BH}} \\
\left(M_{\odot}\right)\end{array}$ & $\begin{array}{l}M_{\mathrm{H}_{2}} \\
\left(M_{\odot}\right)\end{array}$ \\
\hline 3 C 31 & 0.017 & Seyfert & $9.08 \times 10^{23}$ & $<2.0 \times 10^{-4}$ & 3 & $2.4 \times 10^{11}$ & $7.8 \times 10^{7}$ & $(5.1 \pm 0.4) \times 10^{8}$ \\
\hline $3 \mathrm{C} 33$ & 0.060 & Seyfert & $3.95 \times 10^{25}$ & $1.6 \times 10^{-3}$ & 5 & $1.3 \times 10^{11}$ & $4.8 \times 10^{8}$ & $(3.75 \pm 1.5) \times 10^{8}$ \\
\hline $3 \mathrm{C} 98$ & 0.030 & Seyfert & $8.75 \times 10^{24}$ & $3.7 \times 10^{-4}$ & 2 & $7.9 \times 10^{10}$ & $1.7 \times 10^{8}$ & $<7.8 \times 10^{7}$ \\
\hline $3 \mathrm{C} 321$ & 0.097 & Seyfert & $2.6 \times 10^{25}$ & $\ldots$ & 13 & $7.0 \times 10^{11}$ & $\ldots$ & $(3.3 \pm 0.6) \times 10^{9}$ \\
\hline $3 \mathrm{C} 403$ & 0.059 & Seyfert & $3.5 \times 10^{25}$ & $3.3 \times 10^{-3}$ & 5 & $2.4 \times 10^{11}$ & $2.6 \times 10^{8}$ & $(6.6 \pm 1.6) \times 10^{8}$ \\
\hline $3 \mathrm{C} 449$ & 0.017 & Seyfert & $6.51 \times 10^{23}$ & $<7.0 \times 10^{-3}$ & 3 & $2.4 \times 10^{10}$ & $5.1 \times 10^{7}$ & $(1.1 \pm 0.2) \times 10^{8}$ \\
\hline $3 \mathrm{C} 452$ & 0.081 & Seyfert & $7.54 \times 10^{25}$ & $3.3 \times 10^{-3}$ & 13 & $4.5 \times 10^{11}$ & $3.5 \times 10^{8}$ & $8.1 \times 10^{8, c}$ \\
\hline Cen A & 0.0008 & Sey $2^{\mathrm{a}}$ & $5.4 \times 10^{23}$ & $3.0 \times 10^{-5}$ & $\ldots$ & $\ldots$ & $2.0 \times 10^{8}$ & $1.4 \times 10^{8}$ \\
\hline $3 \mathrm{C} 405$ & 0.0565 & Sey $2^{\mathrm{a}}$ & $4.90 \times 10^{27}$ & $8.5 \times 10^{-4}$ & $\cdots$ & $\cdots$ & $2.5 \times 10^{9}$ & $<3.3 \times 10^{8}$ \\
\hline $3 \mathrm{C} 66 \mathrm{~B}$ & 0.022 & LINER & $2.21 \times 10^{24}$ & $<4.4 \times 10^{-5}$ & 3 & $3.0 \times 10^{10}$ & $6.9 \times 10^{8}$ & $<7.8 \times 10^{7}$ \\
\hline $3 \mathrm{C} 84$ & 0.018 & LINER & $3.74 \times 10^{24}$ & $<9.2 \times 10^{-6}$ & $\ldots$ & $\ldots$ & $1.9 \times 10^{9}$ & $(2.14 \pm 0.02) \times 10^{9}$ \\
\hline $3 \mathrm{C} 264$ & 0.022 & LINER & $2.20 \times 10^{24}$ & $<1.8 \times 10^{-5}$ & 13 & $4.4 \times 10^{11}$ & $7.1 \times 10^{8}$ & $(9.3 \pm 1.8) \times 10^{7}$ \\
\hline $3 \mathrm{C} 272.1$ & 0.004 & LINER & $3.1 \times 10^{22}$ & $<8.5 \times 10^{-7}$ & 13 & $2.9 \times 10^{11}$ & $1.5 \times 10^{9}$ & $(9.3 \pm 3.2) \times 10^{5, b}$ \\
\hline $3 \mathrm{C} 274$ & 0.004 & LINER & $3.4 \times 10^{24}$ & $<4.3 \times 10^{-7}$ & 2 & $1.5 \times 10^{11}$ & $2.4 \times 10^{9}$ & $(1.65 \pm 0.15) \times 10^{7}$ \\
\hline $3 \mathrm{C} 296$ & 0.025 & LINER & $1.43 \times 10^{24}$ & $<1.2 \times 10^{-5}$ & 13 & $1.1 \times 10^{12}$ & $1.3 \times 10^{9}$ & $<5.7 \times 10^{7}$ \\
\hline 3C 338 & 0.032 & LINER & $8.63 \times 10^{24}$ & $<2.0 \times 10^{-5}$ & 13 & $1.3 \times 10^{12}$ & $1.7 \times 10^{9}$ & $3 \times 10^{7}$ \\
\hline 3C 388 & 0.091 & LINER & $4.29 \times 10^{25}$ & $2.0 \times 10$ & 9 & 10 人 & $\ldots$ & $<1.2 \times 10^{9, \mathrm{c}}$ \\
\hline $3 \mathrm{C} 465$ & 0.030 & LINER & $6.41 \times 10^{24}$ & $<2.2 \times 10^{-4}$ & 13 & $1.0 \times 10^{12}$ & $2.1 \times 10^{9}$ & $<1.95 \times 10^{7}$ \\
\hline NGC 1265 & 0.027 & LERG $^{\mathrm{a}}$ & $3.39 \times 10^{24}$ & $<6.8 \times 10^{-6}$ & 13 & $1.5 \times 10^{10}$ & $1.0 \times 10^{9}$ & $<5.7 \times 10^{7}$ \\
\hline NGC 6109 & 0.0296 & LERG $^{\mathrm{a}}$ & $1.86 \times 10^{24}$ & & $\ldots$ & $\ldots$ & & $(1.3 \pm 0.3) \times 10^{8}$ \\
\hline NGC 6251 & 0.0244 & LERG $^{\mathrm{a}}$ & $1.20 \times 10^{24}$ & $<2.0 \times 10^{-4}$ & $\ldots$ & $\ldots$ & $6.0 \times 10^{8}$ & $<7.5 \times 10^{7}$ \\
\hline
\end{tabular}

Notes. The first, second, and third columns denote the source, its redshift, and AGN type, respectively. The last was inferred either via optical diagnostic diagrams (see Figure 1) or adopted from NED. The fourth column shows the radio continuum luminosity, adopted from Evans et al. (2006). The fifth column, also adopted from Evans et al. (2006), represents the accretion efficiency (in Eddington units) derived from X-ray observations of the cores of the AGN (the upper limits are obtained assuming $N_{\mathrm{H}}=10^{24}$ atoms $\mathrm{cm}^{-2}$; see Evans et al. 2006 for details). The sixth column shows the stellar age of the source based on fitting stellar population synthesis models to the optical spectra of the sources (encompassing the $\mathrm{H} \alpha$ portion of the spectrum; see Table 5 in Buttiglione et al. 2009). The seventh column shows the stellar mass derived using the 2MASS $K$-band luminosity and stellar age (where available) following Drory et al. (2004; see the text for details). The second to last column shows the BH mass, adopted from Evans et al. (2006), and the last column reports the molecular gas mass obtained from $\mathrm{CO}(1 \rightarrow 0)$ observations (see Table 3) using a conversion factor of $\alpha=M_{\mathrm{H}_{2}} / L_{\mathrm{CO}}^{\prime}=$ $1.5\left(\mathrm{~K} \mathrm{~km} \mathrm{~s}^{-1} \mathrm{pc}^{2}\right)^{-1}$, and assuming $H_{0}=70, \Omega_{M}=0.3$, and $\Omega_{\Lambda}=0.7$. The horizontal lines separate Seyferts (i.e., HERAGN; top) and LINERs (i.e., LERAGN; bottom).

a Based on NED; LERG abbreviates "low-excitation radio galaxy."

b Not considered in our statistical analysis (see Table 3 for details).

c Adopted from Saripalli \& Mack (2007) and scaled to the cosmology used here.

for Research in Millimeter-wave Astronomy (CARMA). We adopt a $\Lambda$ CDM cosmology with $H_{0}=70, \Omega_{M}=0.3$, and $\Omega_{\Lambda}=0.7$.

\section{DATA}

\subsection{Sample}

We here utilize a sample of 21 Type 2 AGNs at $z<0.1$ that have been observed in X-rays (with Chandra or XMMNewton) by Evans et al. (2006). Eighteen out of the 21 AGNs have been drawn from the 3CRR survey, adding three more sources (3C 403, 3C 405, and Cen A) for completeness (see Evans et al. 2006 for details). The sample properties are summarized in Table 1 (see also Table 1 in Evans et al. 2006). We separate our AGNs into LERAGNs (i.e., LINERs) and HERAGNs (i.e., Seyferts) using standard diagnostic tools based on optical emission-line flux ratios where possible (see Figure 1 and Table 1; Kauffmann et al. 2003; Kewley et al. 2001, 2006; Smolčić 2009; Buttiglione et al. 2009). For this we make use of the emission-line fluxes extracted from high-resolution spectroscopy of 3CR sources presented in Buttiglione et al. (2009, 2010, 2011; see also Table 1 in Smolčić 2009). In cases where the relevant emission-line fluxes are not available, we make use of the galaxy-type information available in the NASA
Extragalactic Database (NED) ${ }^{6}$ to separate the sources into LERAGNs and HERAGNs. The sample contains 9 HERAGNs and 12 LERAGNs.

\section{2. $\mathrm{CO}(1 \rightarrow 0)$ Observations and Data Reduction}

At the time of observations, 8 out of the 21 Type 2 AGNs in our sample have already been detected in $\mathrm{CO}(1 \rightarrow 0)$. Thus, we observed the $\mathrm{CO}(1 \rightarrow 0)$ transition line toward the remaining 13 AGNs using the CARMA Interferometer. Observations were performed during Summer 2009 and Spring 2010 for about 4-15 hr per source (Table 2). All targets were observed under good to excellent weather conditions at $3 \mathrm{~mm}$ with 15 antennas (corresponding to 105 baselines) in the two most compact, E and D, configurations (2009 and 2010, respectively). Data on two objects (3C 388 and $3 \mathrm{C} 405$ ) had to be discarded due to technical problems and are excluded in the following. The receivers were tuned to the redshifted $\operatorname{CO}(J=1 \rightarrow 0)$ line frequencies $\left(v_{\text {rest }}=\right.$ 115.2712 GHz; see Table 2 for exact observing frequencies), centering them in the upper sideband. Three bands with 15 channels of $31.25 \mathrm{MHz}$ width each were utilized. The bands were overlapped by two channels to improve calibration of the correlated data set, leading to an effective bandwidth of

\footnotetext{
6 http://nedwww.ipac.caltech.edu
} 


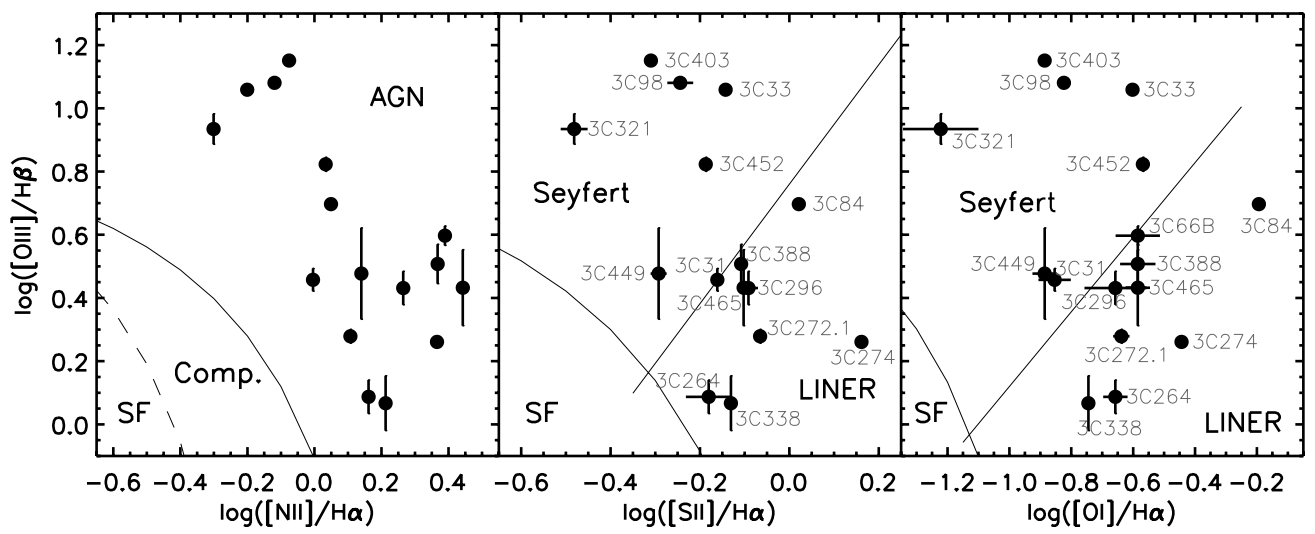

Figure 1. Spectroscopic diagnostic diagrams for our 3CRR (filled dots) sources that separate AGNs into LINERs and Seyferts. The emission-line fluxes have been taken from Buttiglione et al. (2009, see their Table 1). The regions (separated following Kauffmann et al. 2003; Kewley et al. 2001, 2006) that are occupied by star forming (SF), composite (Comp.), and AGN (Seyfert and LINER) galaxies are indicated in the panels. Each 3CRR galaxy is also labeled.

Table 2

Summary of Observations With the CARMA Interferometer

\begin{tabular}{|c|c|c|c|c|c|c|c|}
\hline Source & R.A. (J2000) & Decl. (J2000) & Configuration & $\begin{array}{c}\text { Obs. Freq. } \\
(\mathrm{GHz})\end{array}$ & $\begin{array}{l}\text { On-source } \\
\text { Time (hr) }\end{array}$ & $\begin{array}{c}\text { Beam } \\
(\operatorname{arcsec})\end{array}$ & $\begin{array}{c}\mathrm{rms} / \text { Channel } \\
(\mathrm{mJy})\end{array}$ \\
\hline 3 C 296 & 141652.94 & +104826.50 & $\mathrm{D}$ and $\mathrm{E}$ & 112.460 & 14.1 & $6.4^{\prime \prime} \times 5.5^{\prime \prime}$ & 2.1 \\
\hline $3 \mathrm{C} 321$ & 153143.45 & +240419.10 & $\mathrm{E}$ & 105.079 & 4.0 & $9.8^{\prime \prime} \times 7.2^{\prime \prime}$ & 3.8 \\
\hline $3 \mathrm{C} 33$ & 010852.86 & +132013.80 & $\mathrm{D}$ and $\mathrm{E}$ & 108.746 & 14.8 & $8.7^{\prime \prime} \times 6.3^{\prime \prime}$ & 1.8 \\
\hline $3 \mathrm{C} 403$ & 195215.80 & +023024.47 & E & 108.849 & 5.7 & $9.0^{\prime \prime} \times 6.9^{\prime \prime}$ & 4.7 \\
\hline $3 \mathrm{C} 452$ & 224548.77 & +394115.70 & $\mathrm{D}$ and $\mathrm{E}$ & 106.634 & 15.6 & $6.8^{\prime \prime} \times 5.1^{\prime \prime}$ & 1.8 \\
\hline $3 \mathrm{C} 465$ & 233829.52 & +270155.90 & E & 111.914 & 8.9 & $9.3^{\prime \prime} \times 6.5^{\prime \prime}$ & 3.0 \\
\hline $3 \mathrm{C} 66 \mathrm{~B}$ & 022311.41 & +425931.38 & $\mathrm{E}$ & 112.790 & 4.4 & $8.7^{\prime \prime} \times 6.3^{\prime \prime}$ & 5.1 \\
\hline $3 \mathrm{C} 98$ & 035854.43 & +102603.00 & E & 111.914 & 4.8 & $9.3^{\prime \prime} \times 6.7^{\prime \prime}$ & 3.7 \\
\hline 3C 83.1B (NGC 1265) & 031815.86 & +415127.80 & $\mathrm{D}$ and $\mathrm{E}$ & 112.241 & 13.5 & $4.5^{\prime \prime} \times 3.7^{\prime \prime}$ & 2.1 \\
\hline NGC 6109 & 161740.54 & +350015.10 & $\mathrm{D}$ and $\mathrm{E}$ & 111.957 & 10.6 & $5.0^{\prime \prime} \times 4.0^{\prime \prime}$ & 2.7 \\
\hline NGC 6251 & 163231.97 & +823216.40 & $\mathrm{E}$ & 112.526 & 6.53 & $9.0^{\prime \prime} \times 8.2^{\prime \prime}$ & 3.5 \\
\hline
\end{tabular}

1281.25 MHz (3500 $\mathrm{km} \mathrm{s}^{-1}$ ) per sideband. Phase calibration was performed by observing bright nearby radio quasars every 15 minutes. Bandpass calibration was performed once per track on bright quasars. Fluxes were bootstrapped relative to planets or monitored radio quasars if no planet was available. The total calibration is estimated to be accurate to $15 \%$. Data reduction was performed using the MIRIAD package. The $\mathrm{CO}(1 \rightarrow 0)$ spectra are shown in Figure 2.

\section{RESULTS}

\subsection{CO Data}

$\mathrm{CO}(1 \rightarrow 0)$ has been detected in 4 (3C 33, 3C 321, 3C 403, and NGC 6109) out of the 11 galaxies in our CARMA-CO sample (see Figure 2). To parameterize the emission lines detected in these four galaxies, we fit Gaussian profiles to the line and underlying continuum emission (see Table 3 for line/continuum properties).

Two of our four CO-detected sources (3C 321 and 3C 403) have recently been detected in the $\mathrm{CO}(1 \rightarrow 0)$ transition by Ocaña Flaquer et al. (2010) using the IRAM $30 \mathrm{~m}$ telescope. The line parameters reported by Ocaña Flaquer et al. are in good agreement with ours.

$3 \sigma$ upper limits for $\mathrm{CO}(1 \rightarrow 0)$ non-detections are determined by assuming a line width of $300 \mathrm{~km} \mathrm{~s}^{-1}$, corresponding to the average width of the detected lines. We further complement Table 3 with data from literature for the eight sources with already existing $\mathrm{CO}(1 \rightarrow 0)$ detections, and the two sources (3C 388 and 3C 405) that had to be excluded from our sample.

\subsection{Ancillary Data}

We summarize the physical properties of the 21 sources in our LERAGN and HERAGN sample in Table 1. We adopt the $178 \mathrm{MHz}$ luminosities, accretion efficiencies, and $\mathrm{BH}$ masses from Evans et al. (2006). The stellar ages of our sources, taken from Buttiglione et al. (2009), were derived by fitting stellar population synthesis models to the (H $\alpha$ portion of the sources') optical spectra. Combining the stellar ages with Two Micron All Sky Survey (2MASS) $K$-band luminosities (where available), we computed the stellar masses of our sources following Drory et al. (2004). Drory et al. have parameterized the mass-tolight ratio in $K$ band as a function of stellar age (see their Figure 1) using simple stellar population models (Maraston 1998) and a Salpeter initial mass function. The total systematic uncertainty of such a derived mass-to-light ratio is estimated to be $\sim 25 \%-30 \%$. Finally, from the $\mathrm{CO}(1 \rightarrow 0)$ luminosities inferred for our sources (see Table 3) we estimated the molecular $\left(\mathrm{H}_{2}\right)$ mass using a conversion factor of $\alpha=M_{\mathrm{H}_{2}} / L_{\mathrm{CO}}^{\prime}=1.5(\mathrm{~K}$ $\left.\mathrm{km} \mathrm{s}^{-1} \mathrm{pc}^{2}\right)^{-1}$ (Evans et al. 2005).

We find systematic differences in the average $\mathrm{BH}$ and host galaxy properties of the low- and high-excitation sources (i.e., LINERs and Seyferts, respectively) in our sample (Table 1). This is illustrated in Figure 3, where we also indicate the average properties of our LERAGN and HERAGN, computed 

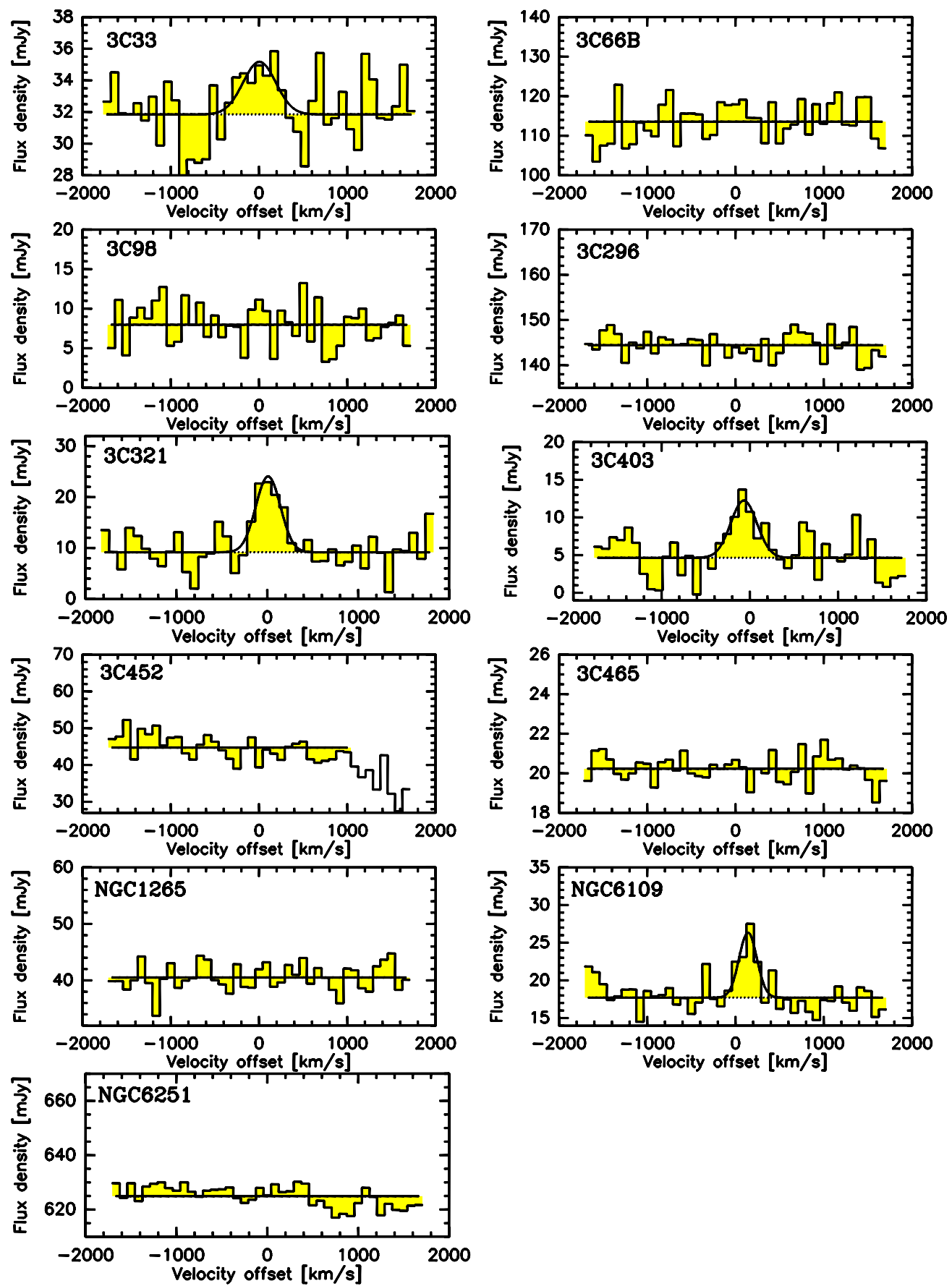

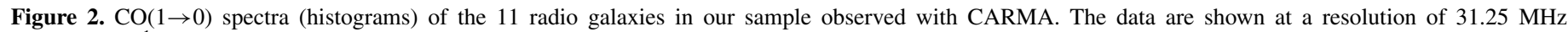
$\left(83-89 \mathrm{~km} \mathrm{~s}^{-1}\right)$. The black curves are Gaussian fits to the line profiles and rest-frame $2.6 \mathrm{~mm}$ continuum emission.

(A color version of this figure is available in the online journal.)

using the ASURV statistical package and assuming log-normal distributions in luminosity and mass. The average properties are specifically given in Table $4 .^{7}$ Compared to LERAGNs, HERAGNs on average have a factor of $\sim 3$ higher radio continuum luminosities, significantly higher accretion efficiencies,

\footnotetext{
7 It should be kept in mind that in ASURV there is an implicit assumption that the censored data follow a similar distribution to that of the measured population. If this is not the case, "average" values calculated by ASURV will be generally biased upward (as our upper limits typically lie toward the bottom end of the distribution). Note however that, if this were the case, it would not change, but only strengthen the results presented here.
}

but about an order of magnitude lower mass central BHs. Furthermore, their host galaxies have about a factor of 1.5 younger stellar populations and stellar masses, but about a factor of $\sim 7$ higher molecular gas masses. As discussed in the next section, this is consistent with the idea that HERAGNs and LERAGNs form two physically distinct populations of galaxies that reflect different phases of massive galaxy formation.

\section{DISCUSSION AND SUMMARY}

Our main result is that HERAGNs have systematically higher molecular gas masses (a factor of $\sim 7$; see Table 4 ), compared to 

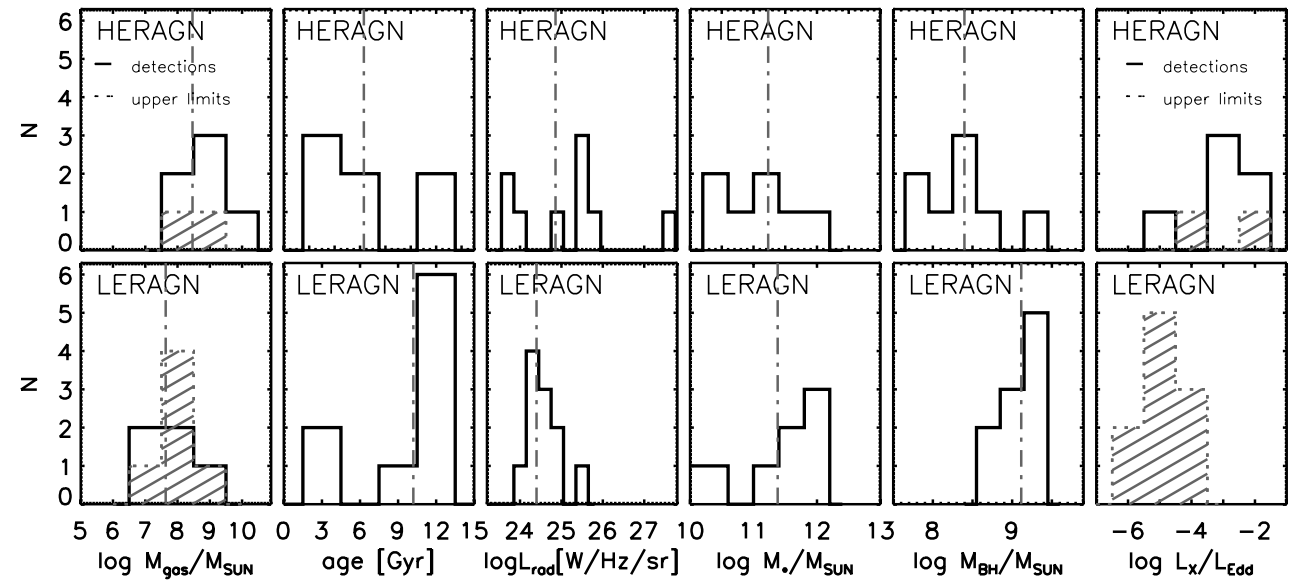

Figure 3. Distribution of physical properties of high- and low-excitation radio AGNs (HERAGNs and LERAGNs, respectively), drawn from Table 1 (excluding the tentative $\mathrm{CO}$ detection in $3 \mathrm{C}$ 272.1). Average values (given in Table 4) are indicated by vertical dot-dashed lines.

Table 3

Molecular $g$ Properties

\begin{tabular}{|c|c|c|c|c|c|c|}
\hline Source & $z$ & $\begin{array}{l}S_{\text {cont }} \\
(\mathrm{mJy})\end{array}$ & $z_{\mathrm{CO}}$ & $\begin{array}{l}\Delta v_{\text {FWHM }} \\
\left(\mathrm{km} \mathrm{s}^{-1}\right)\end{array}$ & $\begin{array}{c}I_{\mathrm{CO}(1 \rightarrow 0)} \\
\left(\mathrm{Jy} \mathrm{km} \mathrm{s}^{-1}\right)\end{array}$ & $\begin{array}{c}L_{\mathrm{CO}}^{\prime} \\
\left(\mathrm{K} \mathrm{km} \mathrm{s}^{-1} \mathrm{pc}^{2}\right)\end{array}$ \\
\hline 3 C 33 & $0.060^{\mathrm{a}}$ & $31.8 \pm 0.4$ & 0.060 & $400 \pm 200$ & $1.5 \pm 0.6$ & $(2.5 \pm 1.0) \times 10^{8}$ \\
\hline $3 \mathrm{C} 66 \mathrm{~B}$ & $0.022^{\mathrm{a}}$ & $113.6 \pm 0.8$ & $\ldots$ & $\ldots$ & $<2.4$ & $<5.2 \times 10^{7}$ \\
\hline 3C 83.1B (NGC 1265) & $0.027^{\mathrm{a}}$ & $40.5 \pm 0.4$ & $\ldots$ & $\ldots$ & $<1.2$ & $<3.8 \times 10^{7}$ \\
\hline $3 \mathrm{C} 98$ & $0.030^{\mathrm{a}}$ & $8.0 \pm 0.4$ & $\ldots$ & $\ldots$ & $<1.3$ & $<5.2 \times 10^{7}$ \\
\hline 3C 296 & $0.025^{\mathrm{a}}$ & $144.4 \pm 0.4$ & $\ldots$ & $\ldots$ & $<1.4$ & $<3.8 \times 10^{7}$ \\
\hline $3 \mathrm{C} 321$ & $0.097^{\mathrm{a}}$ & $9.1 \pm 0.6$ & 0.097 & $320 \pm 70$ & $5.0 \pm 0.9$ & $(2.2 \pm 0.4) \times 10^{9}$ \\
\hline $3 \mathrm{C} 403$ & $0.059^{\mathrm{a}}$ & $4.6 \pm 0.5$ & 0.059 & $350 \pm 100$ & $2.8 \pm 0.7$ & $(4.4 \pm 1.1) \times 10^{8}$ \\
\hline $3 \mathrm{C} 452^{\mathrm{b}}$ & $0.081^{\mathrm{a}}$ & $44.7 \pm 0.6$ & $\ldots$ & $\ldots$ & $\ldots$ & $<5.4 \times 10^{8}$ \\
\hline $3 \mathrm{C} 465$ & $0.030^{\mathrm{a}}$ & $20.2 \pm 0.1$ & $\ldots$ & $\ldots$ & $<0.3$ & $<1.3 \times 10^{7}$ \\
\hline NGC 6109 & $0.0296^{\mathrm{c}}$ & $17.7 \pm 0.3$ & 0.0301 & $230 \pm 50$ & $2.2 \pm 0.4$ & $(8.8 \pm 1.7) \times 10^{7}$ \\
\hline NGC 6251 & $0.0244^{\mathrm{c}}$ & $624.9 \pm 0.6$ & $\ldots$ & $\ldots$ & $<1.9$ & $<5.0 \times 10^{7}$ \\
\hline Cen $A^{d}$ & $0.0008^{\mathrm{c}}$ & $\ldots$ & $\ldots$ & $\ldots$ & $\ldots$ & $9.4 \times 10^{7}$ \\
\hline $3 \mathrm{C} 272.1(\mathrm{M} 84)^{\mathrm{e}, \mathrm{f}}$ & $0.004^{\mathrm{a}}$ & $\ldots$ & 0.0028 & 200 & $1.8 \pm 0.6$ & $(6.2 \pm 2.1) \times 10^{5}$ \\
\hline 3C $274(\mathrm{M} 87)^{\mathrm{e}}$ & $0.004^{\mathrm{a}}$ & $\ldots$ & 0.0035 & 200 & $20 \pm 2$ & $(1.1 \pm 0.1) \times 10^{7}$ \\
\hline $3 C 31^{g}$ & $0.017^{\mathrm{a}}$ & $\ldots$ & 0.0169 & 450 & $27 \pm 2$ & $(3.4 \pm 0.2) \times 10^{8}$ \\
\hline $3 \mathrm{C} 449^{\mathrm{e}}$ & $0.017^{\mathrm{a}}$ & $\ldots$ & 0.0169 & 500 & $6 \pm 1$ & $(7.6 \pm 1.3) \times 10^{7}$ \\
\hline $3 \mathrm{C} 84(\mathrm{NGC} 1275)^{\mathrm{g}}$ & $0.018^{\mathrm{a}}$ & $\ldots$ & 0.0176 & 200 & $104 \pm 1$ & $(1.43 \pm 0.01) \times 10^{9}$ \\
\hline $3 \mathrm{C} 264^{\mathrm{e}}$ & $0.022^{\mathrm{a}}$ & $\ldots$ & 0.02 & 200 & $3.5 \pm 0.7$ & $(6.2 \pm 1.2) \times 10^{7}$ \\
\hline $3 C 338^{h}$ & $0.032^{\mathrm{a}}$ & $\ldots$ & 0.030 & $\ldots$ & 0.495 & $2.0 \times 10^{7}$ \\
\hline $3 \mathrm{C} 405^{\mathrm{g}}$ & $0.0565^{\mathrm{c}}$ & $\ldots$ & $\ldots$ & $\ldots$ & $<1.5$ & $<2.2 \times 10^{8}$ \\
\hline 3C 388 & 0.091 & $\ldots$ & $\ldots$ & $\ldots$ & $\ldots$ & $\ldots$ \\
\hline
\end{tabular}

Notes. The columns show the source, its redshift, the observed continuum flux density $\left(S_{\text {cont }}\right)$, the redshift based on the $\mathrm{CO}(1 \rightarrow 0)$ emission line $\left(z_{\mathrm{CO}}\right)$, the line width at half-maximum $\left(v_{\mathrm{FWHM}}\right)$, the $\mathrm{CO}$ line intensity $\left(I_{\mathrm{CO}(1 \rightarrow 0)}\right)$, and luminosity $\left(L_{\mathrm{CO}}^{\prime}\right.$; see Equation (4) in Evans et al. 2005). For sources in which the $\mathrm{CO}$ line was not detected we report $3 \sigma$ upper limits, computed assuming $\Delta v_{\mathrm{FWHM}}=300 \mathrm{~km} \mathrm{~s}^{-1}$.

${ }^{a}$ Adopted from Buttiglione et al. (2009).

${ }^{\mathrm{b}}$ Due to the strong contribution of complex, steeply sloped millimeter continuum emission from extended radio jets to the millimeter emission of this source, the continuum was fitted over only 33 channels, where the jet contribution is estimated to be small after deconvolution. Due to this uncertainty, however, we do not consider this source in our statistical analysis.

${ }^{\mathrm{c}}$ Adopted from Evans et al. (2006).

d Adopted from Eckart et al. (1990) and scaled to the cosmology used here.

${ }^{\mathrm{e}} z_{\mathrm{CO}}, \Delta v_{\mathrm{FWHM}}$, and $I_{\mathrm{CO}(1 \rightarrow 0)}$ are taken from Ocaña Flaquer et al. (2010). Given that their observations were conducted with the IRAM $30 \mathrm{~m}$ telescope, we take $1 \mathrm{~K}=4.95 \mathrm{Jy}$ and compute $L_{\mathrm{CO}}^{\prime}$ using the cosmology adopted here.

${ }^{\mathrm{f}}$ Tentative detection.

${ }^{\mathrm{g}} z_{\mathrm{CO}}, \Delta v_{\mathrm{FWHM}}$, and $I_{\mathrm{CO}(1 \rightarrow 0)}$ are adopted from Evans et al. (2005). $L_{\mathrm{CO}}^{\prime}$ was computed using the cosmology adopted here.

${ }^{\mathrm{h}} I_{\mathrm{CO}(1 \rightarrow 0)}$ adopted from Leon et al. (2001).

LERAGNs. Ocaña Flaquer et al. (2010) have found a similar trend by dividing their sample $(\sim 50$ radio AGNs observed with the IRAM $30 \mathrm{~m}$ telescope, partially overlapping with our sample) into FR class I and II objects. They find that the molecular gas mass in FR IIs is a factor of $\sim 4$ higher than that in FR Is. The FR class can be taken to roughly correspond to the low- and high-excitation classification. ${ }^{8}$ Ocaña Flaquer et al. (2010) have, however, concluded that the systematic

\footnotetext{
8 Almost all FR I-low power-radio galaxies are LERAGNs, while optical hosts of FR IIs, which are typically more powerful than FR Is (e.g., Fanaroff \& Riley 1974; Ledlow \& Owen 1992), usually have strong emission lines. Note, however, that the correspondence between the FR class and the presence of emission lines is not one-to-one.
} 
Table 4

Average Properties of the $z<0.1$ High- and Low-excitation Radio AGNs

\begin{tabular}{lccccc}
\hline \hline $\begin{array}{l}\text { AGN } \\
\text { Type }\end{array}$ & $\begin{array}{c}L_{178 \mathrm{MHz}} \\
\left(\mathrm{W} \mathrm{Hz}^{-1} \mathrm{sr}^{-1}\right)\end{array}$ & $\begin{array}{c}\text { Stellar Age } \\
(\mathrm{Gyr})\end{array}$ & $\begin{array}{c}M_{*} \\
\left(M_{\odot}\right)\end{array}$ & $\begin{array}{c}M_{\mathrm{BH}} \\
\left(M_{\odot}\right)\end{array}$ & $\begin{array}{c}M_{\mathrm{H}_{2}} \\
\left(M_{\odot}\right)\end{array}$ \\
\hline HERAGN & $(7.2 \pm 4.9) \times 10^{24}$ & $6.3 \pm 1.6$ & $(1.7 \pm 0.7) \times 10^{11}$ & $(2.5 \pm 1.0) \times 10^{8}$ & $(2.9 \pm 1.2) \times 10^{8}$ \\
\hline LERAGN & $(2.5 \pm 1.1) \times 10^{24}$ & $10.2 \pm 1.4$ & $(2.4 \pm 1.4) \times 10^{11}$ & $(1.3 \pm 0.2) \times 10^{9}$ & $(4.3 \pm 1.9) \times 10^{7, \mathrm{a}}$ \\
\hline
\end{tabular}

Note. ${ }^{a}$ The given limit was computed excluding the tentative CO detection in 3C 272.1 (see Table 3). Including the gas mass for this source yields an average of $(1.8 \pm 1.5) \times 10^{7} M_{\odot}$.

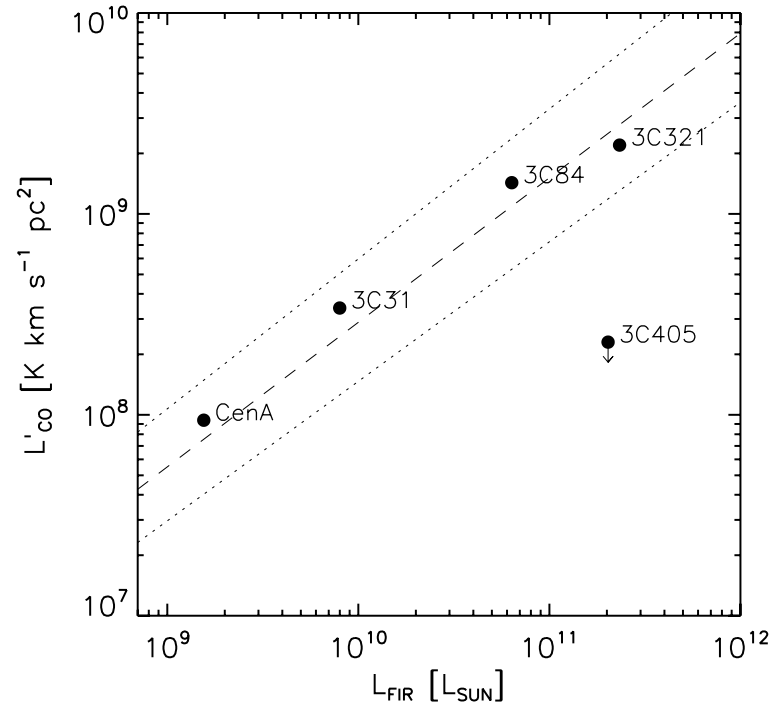

Figure 4. CO vs. FIR luminosity for our local AGN sources detected with IRAS. The lines represent the $L_{\mathrm{CO}}^{\prime}-L_{\mathrm{FIR}}$ correlation derived by Riechers et al. (2006).

differences they find are likely a result of a Malmquist bias, i.e., simply due to a systematically higher redshift of their FR-II sources. Although our HERAGNs lie on average at a slightly higher redshift, compared to our LERAGNs ( 0.046 versus 0.030 , respectively) in the following, we argue that the systematic differences we find in molecular gas mass are not due to a Malmquist bias.

Morić et al. (2010) have shown that the redshift distributions of carefully selected samples of radio-selected LINERs and Seyferts are approximately the same (see their Figure 6). This eliminates Malmquist bias from their results. They find that the detection fraction in the far-infrared (FIR) is significantly lower for LINERs than for Seyferts (6.5\% versus $22 \%$, respectively) in their sample. Assuming that the star formation law parameterized by $L_{\mathrm{CO}}^{\prime}$ (as a proxy for total gas mass) and $L_{\text {FIR }}$ (as a proxy for star formation rate; e.g., Kennicutt 1998; Solomon \& Vanden Bout 2005; Bigiel et al. 2008), on average, correctly represents the star formation properties of these samples (as confirmed by the CO/FIR luminosities of the IRASdetected sources analyzed here; see Figure 4), the lower average FIR luminosity in low-excitation sources (i.e., LINER) implies lower gas masses than in high-excitation (i.e., Seyfert) types of galaxies. A similar result is obtained based on average (optically derived) star formation rates, ${ }^{9}$ suggesting that those in LINERs are by about a factor of three lower than in Seyferts in a redshift-matched sample. These findings suggest that the

\footnotetext{
9 Morić et al. (2010) derived star formation rates for each galaxy in their sample via stellar population synthesis model fitting to the Sloan Digital Sky Survey (SDSS) photometry of the host galaxy (see also Smolčić et al. 2008).
}

systematic differences in molecular gas mass in HERAGNs and LERAGNs are physical, and not due to Malmquist bias.

The systematically higher molecular gas masses that we find in HERAGNs, relative to LERAGNs in our $z<0.1$ radio AGN sample, are in excellent agreement with the systematic differences in various properties of HERAGNs and LERAGNs, both on pc and kpc galaxy scales (see Section 1 and Table 1).

We find that, on average, HERAGNs have lower stellar masses and stellar ages compared to LERAGNs (see Table 4; see also Smolčić 2009). This is consistent with HERAGNs and LERAGNs being green valley and red sequence sources, respectively. Furthermore, we show that HERAGNs have on average higher radio luminosities than LERAGNs, consistent with the results presented in Kauffmann et al. (2008). Kauffmann et al. have shown that the fraction of radio AGN with strong emission lines in their spectra significantly rises beyond $\sim 10^{25} \mathrm{~W} \mathrm{~Hz}^{-1}$. In general, the comparison of the $\mathrm{BH}$ and host galaxy properties inferred for our $21 z<0.1$ AGN with much larger samples of radio AGN (Kauffmann et al. 2008; Smolčić 2009) suggests that our AGN sample is representative of HERAGNs and LERAGNs in the nearby universe.

From the average stellar masses that we infer for our high- and low-excitation sources, we extrapolate that they occupy $\sim 3 \times$ $10^{13} M_{\odot}$ and $\sim 5 \times 10^{14} M_{\odot}$ halos, respectively (e.g., Behroozi et al. 2010; Moster et al. 2010). Compared to the systematic molecular gas mass difference, this yields an even more dramatic discrepancy of more than two orders of magnitude in the average molecular gas fractions in HERAGNs $\left(\sim 10^{-5}\right)$ and LERAGNs $\left(\sim 9 \times 10^{-8}\right)$. The discrepancy remains significant (about an order of magnitude) if the average gas-to-stellar mass fraction (which can be interpreted as star formation efficiency) is considered.

On small scales, the average $\mathrm{BH}$ accretion efficiencies in HERAGNs and LERAGNs suggest different supermassive black hole accretion mechanisms (standard disk accretion of cold gas in HERAGN versus Bondi accretion of hot gas in LERAGN; see Evans et al. 2006; Hardcastle et al. 2006). Furthermore, the higher BH masses in LERAGN suggest a later evolution stage of their host galaxies, compared to that of HERAGN. This is further strengthened by the higher stellar masses in LERAGN, as well as older stellar ages, and less massive gas reservoirs. In the blue-to-red galaxy formation picture, blue gas-rich galaxies are thought to transform into red-and-dead gas-poor galaxies, the stellar populations in the host galaxies of HERAGN are expected to be younger and have lower masses, while their molecular gas reservoirs-fueling further stellar mass growth-are expected to be higher than those in LERAGN. This is in very good agreement with the results presented here. Thus, in summary, our results strengthen the idea that LERAGNs and HERAGNs form two physically distinct galaxy populations that reflect different stages of massive galaxy formation. 
The authors thank F. Bertoldi and K. Knudsen for insightful discussions. The research leading to these results has received funding from the European Union's Seventh Framework program under grant agreement 229517. D.A.R. acknowledges support from NASA through an award issued by JPL/Caltech and from NASA through Hubble Fellowship grant HST-HF51235.01 awarded by the Space Telescope Science Institute, which is operated by the Association of Universities for Research in Astronomy, Inc., for NASA, under contract NAS 5-26555. Support for CARMA construction was derived from the Gordon and Betty Moore Foundation, the Kenneth T. and Eileen L. Norris Foundation, the James S. McDonnell Foundation, the Associates of the California Institute of Technology, the University of Chicago, the states of California, Illinois, and Maryland, and the National Science Foundation. Ongoing CARMA development and operations are supported by the National Science Foundation under a cooperative agreement and by the CARMA partner universities.

\section{REFERENCES}

Allen, S. W., Dunn, R. J. H., Fabian, A. C., Taylor, G. B., \& Reynolds, C. S 2006, MNRAS, 372, 21

Behroozi, P. S., Conroy, C., \& Wechsler, R. H. 2010, ApJ, 717, 379

Bell, E. F., et al. 2004a, ApJ, 608, 752

Bell, E. F., et al. 2004b, ApJ, 600, L11

Bigiel, F., Leroy, A., Walter, F., Brinks, E., de Blok, W. J. G., Madore, B., \& Thornley, M. D. 2008, AJ, 136, 2846

Borch, A., et al. 2006, A\&A, 453, 869

Bower, R. G., Benson, A. J., Malbon, R., Helly, J. C., Frenk, C. S., Baugh, C. M., Cole, S., \& Lacey, C. G. 2006, MNRAS, 370, 645

Brown, M. J. I., Dey, A., Jannuzi, B. T., Brand, K., Benson, A. J., Brodwin, M., Croton, D. J., \& Eisenhardt, P. R. 2007, ApJ, 654, 858

Buttiglione, S., Capetti, A., Celotti, A., Axon, D. J., Chiaberge, M., Duccio Macchetto, F., \& Sparks, W. B. 2009, A\&A, 495, 1033

Buttiglione, S., Capetti, A., Celotti, A., Axon, D. J., Chiaberge, M., Macchetto, F. D., \& Sparks, W. B. 2010, A\&A, 509, A6

Buttiglione, S., Capetti, A., Celotti, A., Axon, D. J., Chiaberge, M., Macchetto, F. D., \& Sparks, W. B. 2011, A\&A, 525, A28
Croton, D. J., et al. 2006, MNRAS, 365, 11

Drory, N., Bender, R., Feulner, G., Hopp, U., Maraston, C., Snigula, J., \& Hill, G. J. 2004, ApJ, 608, 742

Eckart, A., Cameron, M., Rothermel, H., Wild, W., Zinnecker, H., Rydbeck, G., Olberg, M., \& Wiklind, T. 1990, ApJ, 363, 451

Evans, A. S., Mazzarella, J. M., Surace, J. A., Frayer, D. T., Iwasawa, K., \& Sanders, D. B. 2005, ApJS, 159, 197

Evans, D. A., Worrall, D. M., Hardcastle, M. J., Kraft, R. P., \& Birkinshaw, M. 2006, ApJ, 642, 96

Faber, S. M., et al. 2007, ApJ, 665, 265

Fanaroff, B. L., \& Riley, J. M. 1974, MNRAS, 167, 31

Hardcastle, M. J., Evans, D. A., \& Croston, J. H. 2006, MNRAS, 370, 1893

Jackson, N., \& Rawlings, S. 1997, MNRAS, 286, 241

Kauffmann, G., Heckman, T. M., \& Best, P. N. 2008, MNRAS, 384 953

Kauffmann, G., et al. 2003, MNRAS, 341, 33

Kennicutt, R. C., Jr. 1998, ARA\&A, 36, 189

Kewley, L. J., Dopita, M. A., Sutherland, R. S., Heisler, C. A., \& Trevena, J. 2001, ApJ, 556, 121

Kewley, L. J., Groves, B., Kauffmann, G., \& Heckman, T. 2006, MNRAS, 372 , 961

Ledlow, M. J., \& Owen, F. N. 1992, BAAS, 24, 1144

Leon, S., Lim, J., Combes, F., \& van-Trung, D. 2001, in QSO Hosts and Their Environments, ed. I. Márquez et al. (Dordrecht: Kluwer), 185

Maraston, C. 1998, MNRAS, 300, 872

Morić, I., Smolčić, V., Kimball, A., Riechers, D. A., Ivezić, Ž., \& Scoville, N. 2010, ApJ, 724, 779

Moster, B. P., Somerville, R. S., Maulbetsch, C., van den Bosch, F. C., Macciò, A. V., Naab, T., \& Oser, L. 2010, ApJ, 710, 903

Ocaña Flaquer, B., Leon, S., Combes, F., \& Lim, J. 2010, A\&A, 518, A9

Peacock, J. A. 1985, MNRAS, 217, 601

Riechers, D. A., et al. 2006, ApJ, 650, 604

Saripalli, L., \& Mack, K.-H. 2007, MNRAS, 376, 1385

Sijacki, D., \& Springel, V. 2006, MNRAS, 366, 397

Sijacki, D., Springel, V., di Matteo, T., \& Hernquist, L. 2007, MNRAS, 380, 877

Smolčić, V. 2009, ApJ, 699, L43

Smolčić, V., et al. 2008, ApJS, 177, 14

Smolčić, V., et al. 2009, ApJ, 696, 24

Solomon, P. M., \& Vanden Bout, P. A. 2005, ARA\&A, 43, 677

Willott, C. J., Rawlings, S., Blundell, K. M., Lacy, M., \& Eales, S. A. 2001, MNRAS, 322, 536 